\title{
Adequacy of Therapy for People with Both COPD and Heart Failure in the UK: Historical Cohort Study
}

This article was published in the following Dove Press journal: Pragmatic and Observational Research

\author{
Konstantinos Kostikas, (D) \\ Chin Kook Rhee, $\mathbb{D}^{2}$ \\ John R Hurst, ${ }^{3}$ \\ Piergiuseppe Agostoni, (D) ${ }^{4,5}$ \\ Hui Cao, ${ }^{6}$ Robert Fogel, (1D ${ }^{6}$ \\ Rupert Jones, (iD ${ }^{7}$ \\ Janwillem WH Kocks, (D) ${ }^{8-10}$ \\ Karen Mezzi, \\ Simon Wan Yau Ming, \\ Ronan Ryan, ${ }^{8}$ \\ David B Price $\mathbb{I D}^{8,11}$
}

'Novartis Pharma AG, Basel, Switzerland; ${ }^{2}$ Division of Pulmonary, Allergy and Critical Care Medicine, Department of Internal Medicine, Seoul St. Mary's Hospital, College of Medicine, The Catholic University of Korea, Seoul, South Korea; ${ }^{3}$ UCL Respiratory, University College London, London, UK; ${ }^{4}$ Centro Cardiologico Monzino, IRCCS, Milan, Italy; ${ }^{5}$ Department of Clinical Science and Community Health, University of Milan, Milan, Italy; ${ }^{6}$ Novartis Pharmaceuticals Corporation, East Hanover, NJ, USA; ${ }^{7}$ Plymouth University, Faculty of Medicine and Dentistry, Plymouth, UK; ${ }^{8}$ Observational and Pragmatic Research Institute, Singapore, Singapore; ${ }^{9} \mathrm{General}$ Practitioners Research Institute, Groningen, the Netherlands; ${ }^{10}$ Groningen Research Institute for Asthma and COPD (GRIAC), University Medical Center Groningen, Groningen, the Netherlands; "Centre of Academic Primary Care, Division of Applied Health Sciences, University of Aberdeen, Aberdeen, UK

Correspondence: David B Price Centre of Academic Primary Care, Division of Applied Health Sciences, University of Aberdeen, Polwarth Building, Foresterhill, Aberdeen AB25 2ZD, UK

Tel +65 68029724

Email dprice@opri.sg
Purpose: Chronic obstructive pulmonary disease (COPD) and heart failure (HF) often occur concomitantly, presenting diagnostic and therapeutic challenges for clinicians. We examined the characteristics of patients prescribed adequate versus inadequate therapy within 3 months after newly diagnosed comorbid COPD or HF.

Patients and Methods: Eligible patients in longitudinal UK electronic medical record databases had pre-existing HF and newly diagnosed COPD (2017 GOLD groups B/C/D) or preexisting COPD and newly diagnosed HF. Adequate COPD therapy was defined as long-acting bronchodilator(s) with/without inhaled corticosteroid; adequate HF therapy was defined as betablocker plus angiotensin-converting enzyme inhibitor and/or angiotensin receptor blocker.

Results: Of 2439 patients with HF and newly diagnosed COPD (mean 75 years, 61\% men), adequate COPD therapy was prescribed for 726 (30\%) and inadequate for 1031 (42\%); 682 $(28 \%)$ remained untreated for COPD. Adequate (vs inadequate) COPD therapy was less likely for women (35\%) than men (45\%), smokers (36\%) than ex-/non-smokers $(45 \%)$, and non-obese $(41 \%)$ than obese $(47 \%)$; spirometry was recorded for $57 \%$ prescribed adequate versus 35\% inadequate COPD therapy. Of 12,587 patients with COPD and newly diagnosed HF (mean 75 years, 60\% men), adequate HF therapy was prescribed for 2251 (18\%) and inadequate for 5332 (42\%); $5004(40 \%)$ remained untreated for HF. Adequate (vs inadequate) HF therapy was less likely for smokers (27\%) than ex-/non-smokers (32\%) and nonobese (30\%) than obese (35\%); spirometry was recorded for $65 \%$ prescribed adequate versus $39 \%$ inadequate HF therapy.

Conclusion: Many patients with comorbid COPD/HF receive inadequate therapy after new diagnosis. Improved equity of access to integrated care is needed for all patient subgroups. Keywords: beta-blocker, long-acting bronchodilator, integrated care, multimorbidity

\section{Plain Language Summary}

The ageing of populations globally has brought attention to the issue of multimorbidity as a common concern and area for prioritization of research. In older individuals, COPD and heart failure (HF) often occur concomitantly, presenting diagnostic and therapeutic challenges for clinicians. However, published guidance on the treatment of comorbid COPD and $\mathrm{HF}$ is sparse, as consensus guidelines focus on the treatment of individual conditions. We used well-managed, longitudinal medical record databases in the UK to examine the demographic and clinical characteristics of patients prescribed adequate versus inadequate therapy within 3 months after newly diagnosed comorbid COPD or HF.

We observed low levels of guidelines-recommended prescribing for therapy of new COPD and new HF diagnoses among patients with comorbid COPD and HF. Only one- 
third $(30 \%)$ of patients in the HF-new COPD cohort were prescribed adequate COPD therapy within 3 months of the COPD diagnosis, and less than one-fifth (18\%) of patients in the COPDnew HF cohort were prescribed adequate HF therapy within 3 months of the HF diagnosis. Patients most likely to be prescribed inadequate COPD therapy included women, current smokers, and non-obese patients, and those most likely to be prescribed inadequate HF therapy included current smokers and non-obese patients.

Further work is needed to understand the reasons for underprescribing and to address them. In addition, readily accessible clinical guidance is needed that addresses not only the diagnosis but the care of patients with comorbid COPD and HF. Finally, improved equity of access to integrated care for all patient subgroups with comorbid COPD and HF is needed, particularly for women, smokers, and non-obese patients.

\section{Introduction}

The ageing of populations globally has brought attention to the issue of multiple morbidities as a common concern and area for prioritization of research. ${ }^{1-3}$ In older individuals, chronic obstructive pulmonary disease (COPD) and heart failure (HF) often occur concomitantly. ${ }^{4,5}$ The reported prevalence of $\mathrm{HF}$ in patients with COPD ranges from $20 \%$ to $70 \%,{ }^{4}$ with 2.6 times greater odds of HF in patients with COPD versus without COPD calculated in a recent metaanalysis, ${ }^{6}$ and the presence of COPD is a significant risk factor for $\mathrm{HF}$, with relative risks ranging from 1.5 to 6.8 , depending on age, reported in a large population-based study in the UK. ${ }^{7}$ The reported prevalence of COPD in patients with $\mathrm{HF}$ ranges from $9 \%$ to $52 \%{ }^{8}$

Patients with comorbid COPD and HF have a worse long-term prognosis than those with either condition alone. ${ }^{9}$ The presence of COPD is a predictor of hospitalization and death from cardiovascular events, ${ }^{10-13}$ while the presence of HF increases the risk of death in patients with COPD. ${ }^{14,15}$ However, patients with comorbid COPD and HF present both diagnostic and therapeutic challenges for clinicians. Overlapping signs and symptoms and similar risk factors, such as smoking, older age, and inactivity, contribute to the diagnostic challenge, ${ }^{16-18}$ particularly as both COPD and HF cause breathlessness. ${ }^{19}$

The focus of published guidelines and management recommendations tends to be on single conditions, thus on either COPD or HF, rather than on the optimal management of comorbid COPD and HF, although the need to treat comorbidities on their merit is increasingly acknowledged. ${ }^{4,18,20-23}$ For example, the Global initiative for chronic Obstructive Lung Disease (GOLD) strategy documents since 2011 have noted that there is no evidence either to treat HF differently for patients with comorbid COPD nor to treat COPD differently in the presence of $\mathrm{HF}^{4}$ Similarly, according to the European Society of Cardiology guidelines since 2008, beta-blockers are not contraindicated for $\mathrm{HF}$ in patients with COPD, although cardioselective beta-blockers are recommended over nonselective beta-blockers. ${ }^{23,24}$

Nonetheless, in light of the opposing pharmacological effects of beta-blockers and beta-agonists, there are concerns that health-care providers may be reluctant to prescribe longacting bronchodilators (LABD), in particular long-acting beta-agonists (LABAs), to treat COPD for patients with comorbid HF. ${ }^{16}$ Also, there is evidence that health-care providers are reluctant to prescribe beta-blockers to treat $\mathrm{HF}$ in patients with comorbid COPD. ${ }^{25,26}$ Many gaps remain in our understanding of the optimal management of patients with comorbid COPD and $\mathrm{HF}^{9}$ and a better understanding of reallife treatment and factors affecting treatment adequacy for these patients is needed.

As part of a broader program to look at current treatment and outcomes for patients with comorbid COPD and HF, our objectives in this historical cohort study were to describe and contrast the demographic and clinical characteristics of 1) patients with pre-existing HF and a new diagnosis of COPD who were prescribed adequate versus inadequate COPD therapy and 2) patients with pre-existing COPD and a new diagnosis of HF who were prescribed adequate versus inadequate HF therapy according to guidelines.

\section{Methods}

\section{Data Sources}

We used two large, well-established UK databases containing anonymized, longitudinal medical record data for this study: the Clinical Practice Research Datalink $(\mathrm{CPRD})^{27}$ and the Optimum Patient Care Research Database (OPCRD). ${ }^{28}$ Both databases contain long-term electronic medical record (EMR) data and are frequently used for pharmacoepidemiological research.

The CPRD is a computerized primary care database containing de-identified, longitudinal data from more than 600 subscribing general practices throughout the UK, representing about 5 million patients with active medical records or $7 \%$ of the UK population. ${ }^{27} \mathrm{~A}$ practice-based quality marker, the "up-to-standard date," is generated by the CPRD for each subscribing practice, and data after the practice up-to-standard date are considered to be 
acceptable, research quality, prospectively recorded data. The CPRD is a governmental, not-for-profit research service, jointly funded by the NHS National Institute for Health Research (NIHR) and the Medicines and Healthcare products Regulatory Agency (MHRA), a part of the Department of Health.

The OPCRD comprises data captured for clinical service evaluation by Optimum Patient Care, a not-for-profit organization that offers free respiratory clinical evaluations with the aim of improving patient outcomes through medical research and services, providing evidence-based recommendations to UK general practices through bespoke software and practice reports. ${ }^{28}$ At the time of the study, the OPCRD included anonymous, longitudinal medical records for over 2.8 million patients from more than 550 primary care practices across the UK. The use of the OPCRD for research purposes has approval from the NHS Health Research Authority and is governed by the Anonymous Data Ethics Protocols and Transparency (ADEPT) committee, the independent scientific advisory committee for the OPCRD.

The CPRD and OPCRD datasets used in this study were first constructed separately, and then, because a small number of family practices contribute data to both databases, we excluded duplicate patients by checking for overlap in key demographics (such as year of birth and sex), blood test results, and test dates before pooling for the analyses, as previously described. ${ }^{29}$

The study was registered with The European Union electronic Register of Post-Authorisation Studies (EUPAS12987) $^{30}$ and was conducted according to recommended procedures for observational research. ${ }^{31,32}$ Research governance approval was obtained from each database for this study (CPRD ISAC reference number 16_039 and ADEPT Approval Reference PROTOCOL1116). Patient data remained anonymized throughout the analyses.

\section{Data Statement}

The datasets supporting the conclusions of this article were derived from the Clinical Practice Datalink (http://www. cprd.com) and the Optimum Patient Care Research Database (http://opcrd.co.uk/). We do not have permission to give public access to these datasets; however, researchers may request access for their own purposes.

\section{Study Design and Patients}

We conducted two descriptive cohort analyses drawing on mutually exclusive, combined datasets drawn from the
CPRD and OPCRD. The first analysis examined and compared the characteristics of patients with pre-existing HF and a new COPD diagnosis who were prescribed adequate versus inadequate COPD therapy within the first 3 months after the COPD diagnosis. The second analysis examined and compared the characteristics of patients with pre-existing COPD and a new HF diagnosis who were prescribed adequate versus inadequate HF therapy within the first 3 months after the HF diagnosis. The study period was 1988 through May 2015, and the "index date" was defined as 3 months after the new COPD or new HF diagnosis.

To be eligible for this study, patients had to have a diagnosis of HF recorded at any time followed by a new COPD diagnosis recorded at any time after the first HF diagnosis (HF-new COPD cohort) or the converse, namely, a recorded COPD diagnosis followed by a new HF diagnosis (COPD-new HF cohort). The diagnoses of COPD and HF were identified by recorded diagnostic Read codes, the coding system used by the UK National Health Service (NHS), ${ }^{33}$ as specified by the NHS Quality and Outcomes Framework (QOF), which provides incentives for key measures to be recorded in primary care EMRs. ${ }^{34,35}$ The inclusion of COPD as a clinical area was effected at the time of QOF implementation in 2004/2005, and HF entered QOF in 2006/2007.

To be eligible, patients were 40 years or older at 3 months after the new diagnosis (COPD or HF, respectively, depending on the cohort). We required that they had continuous, recorded practice data during a total of 2 years plus 3 months, including a baseline year beginning 1 year before the new COPD or new HF diagnosis and an outcome year beginning 3 months after the new COPD diagnosis or new HF diagnosis, respectively. Patients who died during the outcome year were not excluded.

Patients in the HF-new COPD cohort were limited to those with COPD designated as GOLD group B, C, or D (2017 criteria $\left.^{36}\right)$. The GOLD group was determined using exacerbation and $\mathrm{mMRC}$ data recorded closest to and within 5 years before or after the index date. We excluded patients in GOLD group A because their symptoms can be mild enough such that minimal therapy is required and adequacy of therapy could be difficult to judge. In addition, patients were excluded if they had any prior asthma, resolved asthma, or other chronic respiratory diagnoses before the COPD diagnosis. Because the actual date of a diagnosis may occur slightly earlier than when first recorded, the date of COPD diagnosis was set to the first prescription of an LABD if that 
prescription was in the month preceding the first COPD Read code. It was not possible to use a similar definition for HF because of the lack of a HF-specific drug; however, patients in the COPD-new HF cohort had to have recorded evidence of COPD or HF therapy.

\section{Endpoint Definitions}

Patients were assigned to an adequate, inadequate, or notherapy group based on primary care prescribing within the first 3 months after the new diagnosis. We then compared the demographic and clinical characteristics of patients who were prescribed adequate versus inadequate therapy for a new COPD diagnosis (HF-new COPD cohort) or for a new HF diagnosis (COPD-new HF cohort).

Adequate therapy for COPD in GOLD groups B, C, and $\mathrm{D}$ was defined as one or more prescriptions for an LABD, including a LABA, a long-acting muscarinic antagonist (LAMA), or LABA plus LAMA, with or without ICS, while inadequate therapy for COPD was defined as prescription(s) for only short-acting bronchodilators (SABDs). 5,20,36 No COPD therapy was defined as no SABD or LABD prescription.

For HF, adequate therapy was defined as one or more prescriptions for a beta-blocker plus an angiotensinconverting enzyme (ACE) inhibitor and/or an angiotensin receptor blocker $(\mathrm{ARB}) .^{21-23}$ Inadequate therapy for $\mathrm{HF}$ was defined as an ARB plus ACE inhibitor (no betablocker) or monotherapy with a beta-blocker, ARB, or ACE inhibitor. No therapy was defined as no recorded prescription for any of these three drug classes.

\section{Statistical Analyses}

We conducted descriptive analyses of patient demographics, comorbidities, and indicators of disease severity, summarizing categorical data as number (percentage) and continuous and count data as mean (SD) or median (interquartile range [IQR]), as appropriate. For baseline variables with missing data, the percentages of patients with available data were noted.

Baseline demographic and clinical characteristics of patients prescribed adequate versus inadequate COPD therapy after a new COPD diagnosis (with pre-existing HF) were tabulated together, and demographic and clinical characteristics of those prescribed adequate versus inadequate HF therapy after a new HF diagnosis (with preexisting COPD) were tabulated together. Comparisons between adequate and inadequate therapy groups were made using the Mann-Whitney $U$-test or $\chi^{2}$, as appropriate, with p-values.

Analyses were performed using SAS version 9.3 (SAS Institute, Cary, NC).

\section{Results}

\section{Pre-Existing HF and New Diagnosis of COPD: Adequate versus Inadequate COPD Therapy}

A total of 2439 eligible patients with pre-existing HF and a new COPD diagnosis (GOLD groups $\mathrm{B}, \mathrm{C}$, or $\mathrm{D}$ ) were identified from the CPRD and OPCRD (Figure 1). Overall, the mean patient age was 75 years, and $61 \%$ of patients were male. By the end of 3 months after the new COPD diagnosis (the index date), 726 patients $(30 \%)$ had been prescribed adequate therapy for COPD, as per the study definition of prescription(s) for $\operatorname{LABD}(s) \pm$ ICS; 1031 patients $(42 \%)$ were inadequately treated (prescribed only SABDs); and 682 patients $(28 \%)$ remained untreated (no $\mathrm{SABD}$ or LABD prescription).

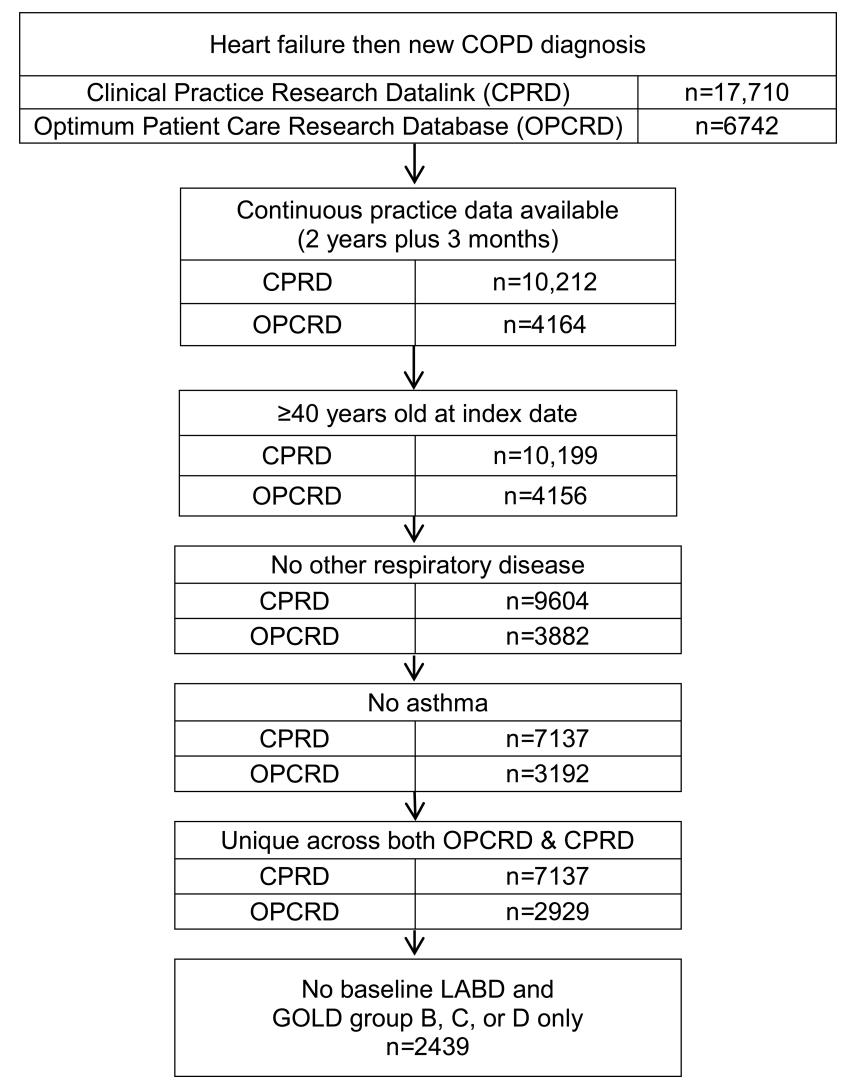

Figure I Flow chart depicting selection of patients with heart failure and new COPD diagnosis.

Abbreviations: CPRD, Clinical Practice Research Datalink; GOLD, Global initiative for chronic Obstructive Lung Disease; LABD, long-acting bronchodilator; OPCRD, Optimum Patient Care Research Database. 
The mean patient age was 75 years in both adequate and inadequate therapy groups, and approximately half of patients in each group were identified as having COPD in GOLD group B, one-third in GOLD C, and one-fifth in GOLD D (Table 1). In the side-by-side comparison between adequate and inadequate therapy groups (Figure 2), the subgroups of patients who were proportionately less likely to be prescribed adequate COPD therapy included women $(35 \%$ prescribed adequate COPD therapy vs $45 \%$ of men), current smokers ( $36 \%$ vs $46 \%$ and $43 \%$ of ex- and non-smokers, respectively), and non-obese patients ( $41 \%$ vs $47 \%$ of obese patients). In addition, of the patients prescribed adequate therapy for new COPD, $57 \%$ had recorded spirometry, while of those prescribed inadequate therapy for new COPD, only $35 \%$ had recorded spirometry. Of the patients with severe breathlessness (mMRC score of 3 or 4 ), $46 \%$ (232/504) were prescribed adequate COPD therapy as compared with $39 \%(494 / 1253)$ of those with mMRC score of 0-2 (Table 1). The median year of the index date was earlier for patients prescribed inadequate COPD therapy (2004 vs 2009 for adequate therapy).

A higher percentage of patients had other common comorbidities in the adequate therapy group as compared with the inadequate therapy group (Table 1).

\section{Pre-Existing COPD and New Diagnosis of HF: Adequate versus Inadequate HF Therapy}

A total of 12,587 eligible patients with pre-existing COPD and new HF diagnosis were identified (Figure 3). Overall baseline patient characteristics were similar to those of the patients with pre-existing HF and new COPD diagnosis: the mean age was 75 years, and $60 \%$ were male. At the end of 3 months after the new HF diagnosis, 2251 (18\%) were prescribed adequate therapy for HF, as per the study definition of prescription(s) for a beta-blocker plus an ACE inhibitor and/ or an ARB; 5332 patients (42\%) were prescribed inadequate therapy (any other combination of these drug classes); and 5004 patients $(40 \%)$ remained untreated.

There were differences in sex ratios and mean ages of patients in adequate versus inadequate therapy groups; however, these differences were not numerically large, with mean ages 73.3 versus 74.5 years, respectively, and as $28 \%$ of women and $31 \%$ of men received adequate HF therapy. Other differences between adequate versus inadequate HF therapy groups were numerically larger (Table 2). For
Table I Demographic and Clinical Characteristics of Patients with Pre-Existing Heart Failure (HF) Who Were Prescribed Adequate versus Inadequate COPD Therapy Within 3 Months of a New COPD Diagnosis (HF-New COPD Cohort)

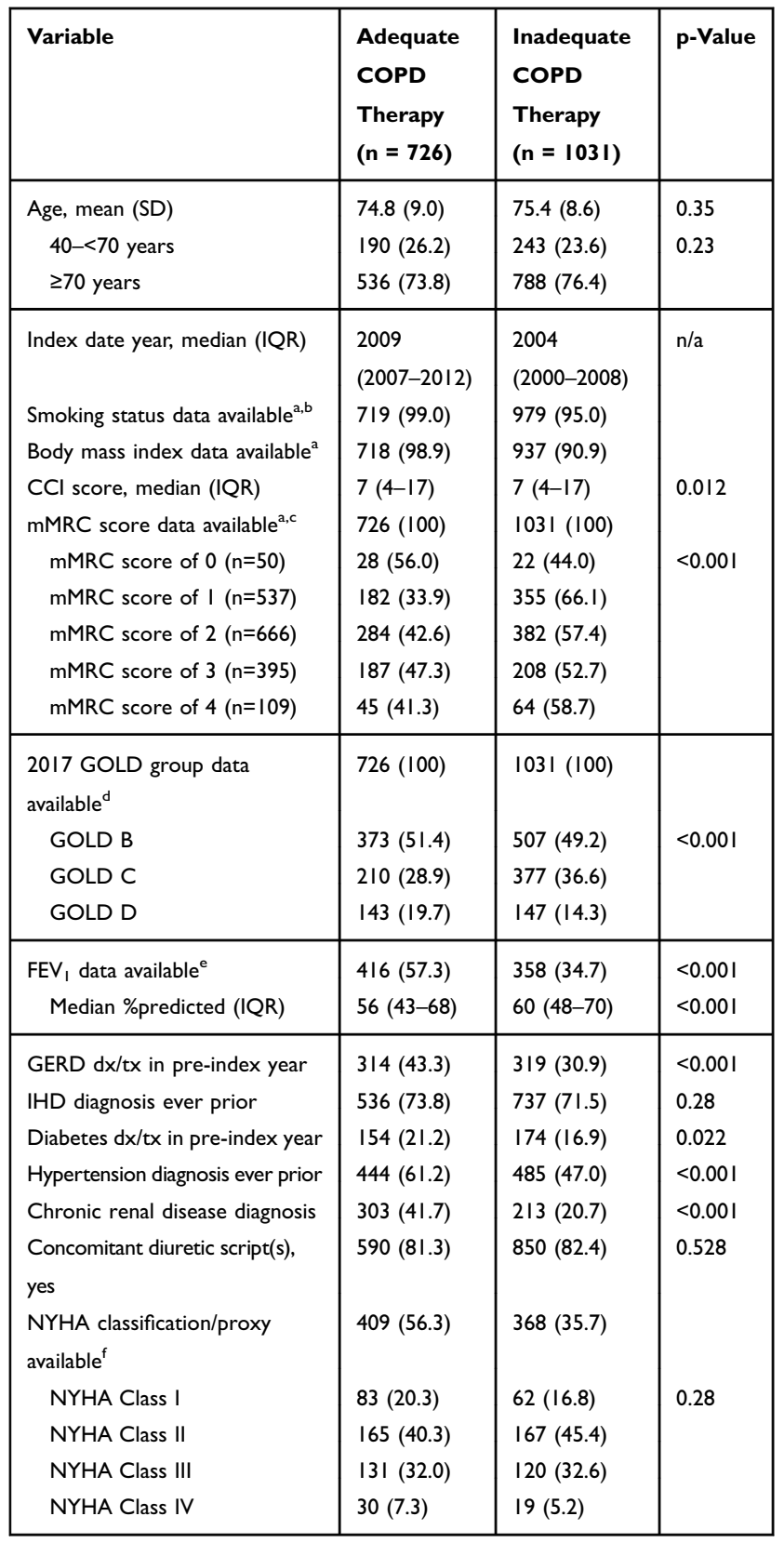

Notes: Data are presented as $\mathrm{n}(\%)$ unless otherwise indicated. Comparisons made using $\chi^{2}$ test for categorical values and Mann-Whitney U-test for continuous or count variables. ${ }^{\text {a }}$ Patient percentages for $\mathrm{mMRC}$ are presented as row percentages; the other variables are calculated using column percentages. ${ }^{b}$ Smoking status was determined using the Read code closest to and $<5$ years before the index date ( 3 months after new COPD diagnosis). ${ }^{\mathrm{m}} \mathrm{mMRC}$ score was defined as the Read code recorded closest to and $<5$ years before (or otherwise $<5$ years after) the index date. ${ }^{\mathrm{d}} \mathrm{GOLD}$ group was calculated using exacerbation and mMRC data recorded closest to and within 5 years before or after the index date $\left(2017\right.$ criteria $\left.^{36}\right) .{ }^{e} \mathrm{FEV}$, determined closest to and within 5 years before the index date. ${ }^{f} \mathrm{NYHA}$ classification determined by Read code closest to and within 5 years before the index date. NYHA data were limited in primary care medical records so breathlessness Read codes were used as proxies for NYHA class.

Abbreviations: $\mathrm{CCl}$, Charlson comorbidity index; GERD, gastroesophageal reflux disease; GOLD, Global initiative for chronic Obstructive Lung Disease; IHD, ischemic heart disease; IQR, interquartile range; mMRC, modified Medical Research Council dyspnea scale; n/a, not available; NYHA, New York Heart Association; script, prescription. 


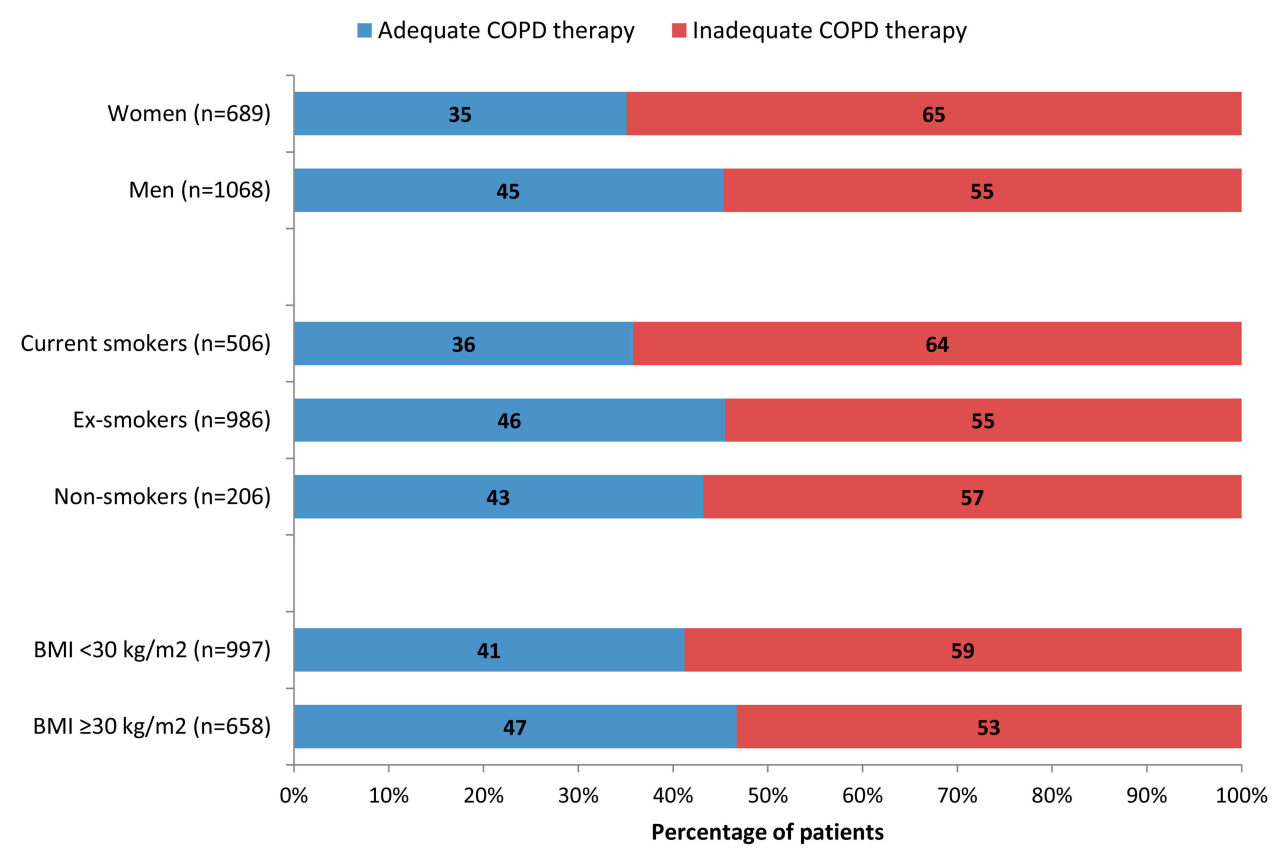

Figure 2 Sex, smoking status, and body mass index categories of patients with pre-existing heart failure (HF) who were prescribed adequate versus inadequate COPD therapy within 3 months of a new COPD diagnosis (HF-new COPD cohort). $\chi^{2}$ test $p \leq 0.001$ for comparisons between women and men and among smoking status categories; $\mathrm{p}=0.016$ for comparisons between BMI categories. Smoking status was determined using the Read code closest to and $<5$ years before the index date $(3$ months after new COPD diagnosis).

example, $27 \%$ of current smokers versus $32 \%$ of ex-smokers and $32 \%$ of non-smokers, and $30 \%$ of non-obese versus $35 \%$ of obese patients were prescribed adequate HF therapy (Figure 4).Of those prescribed adequate HF therapy, 65\% had recorded spirometry versus only $39 \%$ of those receiving inadequate therapy for new HF diagnosis. Of the patients with severe breathlessness (mMRC score of 3 or 4 ), 34\% (377/ 1123 ) were prescribed adequate $\mathrm{HF}$ therapy as compared with $35 \%(1580 / 4480)$ with an mMRC score of $0-2$. As for the HF-new COPD cohort, the median year of the index date was earlier for patients prescribed inadequate HF therapy (2004 vs 2010 for adequate therapy).

Also, similar to the HF-new COPD cohort, among patients with COPD and newly diagnosed HF, other common comorbidities were more frequent in the adequate therapy group as compared with the inadequate therapy group (Table 2).

\section{Discussion}

We identified low levels of guidelines-recommended prescribing for therapy of new COPD and new HF diagnoses among UK patients with comorbid COPD and HF in this historical cohort study. Only one-third (30\%) of patients in the HF-new COPD cohort were prescribed adequate COPD therapy within 3 months of the COPD diagnosis, and less than one-fifth (18\%) of patients in the COPD-new HF cohort were prescribed adequate HF therapy within 3 months of the HF diagnosis. Women, current smokers, and non-obese patients were proportionately less likely to be prescribed adequate COPD therapy, whilst current smokers and non-obese patients were less likely to receive adequate HF therapy.

We broadly defined adequate therapy for COPD (excluding GOLD group $\mathrm{A}$ ) as prescription(s) for $\operatorname{LABD}(\mathrm{s})$ with or without ICS, per GOLD guidelines. ${ }^{4,5}$ In a prior study of COPD prescribing (comorbid HF not assessed), factors that influenced the initial prescription in COPD were comorbid asthma, exacerbation history, symptoms, and lung function. ${ }^{37}$ Breathlessness is a common finding in patients even with stable HF and COPD, ${ }^{19}$ and the presence of dyspnea may encourage prescribing of bronchodilators. However, the fact that some patient subgroups with newly diagnosed COPD and pre-existing HF (eg, women, current smokers, non-obese patients) were less likely to be prescribed adequate COPD therapy in the form of LABDs suggests a need to focus clinical education on COPD therapy for these subgroups. In addition, a large proportion of patients who were prescribed inadequate therapy had no recorded spirometry, although we note that spirometry is often under-recorded in the community setting. ${ }^{38,39}$

With regard to prescribing of adequate HF therapy (betablocker plus an ACE inhibitor and/or an ARB), our findings are in line with prior observational study findings of a reluctance to prescribe beta-blockers to patients with 


\begin{tabular}{|c|r|}
\hline \multicolumn{2}{|c|}{ COPD then new heart failure (HF) diagnosis } \\
\hline Clinical Practice Research Datalink (CPRD) & $\mathrm{n}=30,230$ \\
\hline Optimum Patient Care Research Database (OPCRD) & $\mathrm{n}=11,471$ \\
\hline
\end{tabular}

\begin{tabular}{|c|c|}
\hline & \\
\hline \multicolumn{2}{|c|}{$\begin{array}{l}\text { Continuous practice data available } \\
\text { ( } 2 \text { years plus } 3 \text { months) }\end{array}$} \\
\hline CPRD & $n=15,297$ \\
\hline OPCRD & $n=6551$ \\
\hline \multicolumn{2}{|c|}{$\downarrow$} \\
\hline \multicolumn{2}{|c|}{$\geq 40$ years old at index date } \\
\hline CPRD & $\mathrm{n}=15,286$ \\
\hline OPCRD & $n=6536$ \\
\hline \multicolumn{2}{|c|}{$\downarrow$} \\
\hline \multicolumn{2}{|c|}{ No other respiratory disease } \\
\hline CPRD & $\mathrm{n}=14,485$ \\
\hline OPCRD & $n=6168$ \\
\hline \multicolumn{2}{|c|}{$\downarrow$} \\
\hline \multicolumn{2}{|c|}{ No asthma } \\
\hline CPRD & $\mathrm{n}=9652$ \\
\hline OPCRD & $n=4782$ \\
\hline \multicolumn{2}{|c|}{$\downarrow$} \\
\hline \multicolumn{2}{|c|}{ Unique across both OPCRD \& CPRD } \\
\hline CPRD & $\mathrm{n}=9652$ \\
\hline OPCRD & $\mathrm{n}=4318$ \\
\hline \multicolumn{2}{|c|}{$\downarrow$} \\
\hline \multicolumn{2}{|c|}{$\begin{array}{l}\text { Evidence of COPD or HF treatment at } \\
\text { some time } \\
n=12,587\end{array}$} \\
\hline
\end{tabular}

Figure 3 Flow chart depicting selection of patients with COPD and new heart failure diagnosis.

Abbreviations: CPRD, Clinical Practice Research Datalink; GOLD, Global initiative for chronic Obstructive Lung Disease; LABD, long-acting bronchodilator; OPCRD, Optimum Patient Care Research Database.

comorbid COPD and HF. ${ }^{12,25}$ Even for patients hospitalized for HF, those with comorbid COPD (vs no comorbid COPD) are reportedly less likely to be prescribed beta-blockers and ACE inhibitors/ARBs at the time of discharge from the hospital. ${ }^{12,39}$ In our study, current smokers and non-obese patients were less likely to receive adequate HF therapy, and again we found no recorded spirometry within the prior 5 years for a large proportion of patients $(61 \%)$ with preexisting COPD who were then prescribed suboptimal HF therapy after a new HF diagnosis.

Much of prior work pertains to patients with comorbid COPD and HF who experience COPD exacerbations or are hospitalised. ${ }^{38-41}$ The results of this study add to the limited information available on prescribing practices for patients cared for primarily outside of the hospital setting ${ }^{25,39,42}$ and may potentially raise awareness of the optimal treatment of patients with COPD and HF. The data contained in the CPRD and OPCRD largely pertain to people treated in general practice, as few UK primary care patients with COPD attend
Table 2 Demographic and Clinical Characteristics of Patients with Pre-Existing COPD Who Were Prescribed Adequate versus Inadequate Heart Failure (HF) Therapy Within 3 Months of a New HF Diagnosis (COPD-New HF Cohort)

\begin{tabular}{|c|c|c|c|}
\hline Variable & $\begin{array}{l}\text { Adequate } \\
\text { HF } \\
\text { Therapy (n } \\
=225 \mathrm{I})\end{array}$ & $\begin{array}{l}\text { Inadequate } \\
\text { HF Therapy } \\
(n=5332)\end{array}$ & p-Value \\
\hline $\begin{array}{l}\text { Age, mean (SD) } \\
40-<70 \text { years } \\
\geq 70 \text { years }\end{array}$ & $\begin{array}{l}73.3(9.0) \\
753(33.5) \\
1498(66.5)\end{array}$ & $\begin{array}{l}74.5(8.7) \\
1444(27.1) \\
3888(72.9)\end{array}$ & $\begin{array}{l}<0.001 \\
<0.001\end{array}$ \\
\hline $\begin{array}{l}\text { Index date year, median (IQR) } \\
\text { Smoking status data available }{ }^{a, b} \\
\text { Body mass index data available }{ }^{a} \\
\text { CCl score, median (IQR) } \\
\text { mMRC score data available }{ }^{a, c} \\
\text { mMRC score of } 0(n=332) \\
\text { mMRC score of I }(n=2840) \\
\text { mMRC score of } 2(n=1308) \\
\text { mMRC score of } 3 \quad(n=86 I) \\
\text { mMRC score of } 4 \quad(n=262)\end{array}$ & $\begin{array}{l}2010 \\
(2006-2012) \\
2192(97.4) \\
2199(97.7) \\
13(13-18) \\
1957(86.9) \\
183(55.1) \\
882(31.1) \\
515(39.4) \\
302(35.1) \\
75(28.6)\end{array}$ & $\begin{array}{l}2004 \\
(2000-2008) \\
4925(92.4) \\
4773(89.5) \\
13(13-17) \\
3646(68.4) \\
149(44.9) \\
1958(68.9) \\
793(60.6) \\
559(64.9) \\
187(71.4)\end{array}$ & $\begin{array}{l}<0.001 \\
<0.001\end{array}$ \\
\hline $\begin{array}{l}2017 \text { GOLD group data } \\
\text { available }^{\text {d }} \\
\text { GOLD A } \\
\text { GOLD B } \\
\text { GOLD C } \\
\text { GOLD D }\end{array}$ & $\begin{array}{l}1957(86.9) \\
700(35.8) \\
579(29.6) \\
365(18.7) \\
313(16.0)\end{array}$ & $\begin{array}{l}3646(68.4) \\
1299(35.6) \\
937(25.7) \\
808(22.2) \\
602(16.5)\end{array}$ & 0.002 \\
\hline $\begin{array}{l}\mathrm{FEV}_{\text {, data available }}{ }^{\mathrm{e}} \\
\text { Median \%predicted (IQR) }\end{array}$ & $\begin{array}{l}1470(65.3) \\
59(46-71)\end{array}$ & $\begin{array}{l}2087(39.1) \\
54(41-67)\end{array}$ & $\begin{array}{l}<0.001 \\
<0.001\end{array}$ \\
\hline $\begin{array}{l}\text { GERD dx/tx in pre-index year } \\
\text { IHD diagnosis ever prior } \\
\text { Diabetes dx/tx in pre-index year } \\
\text { Hypertension diagnosis ever prior } \\
\text { Chronic renal disease diagnosis } \\
\text { Concomitant diuretic script(s) } \\
\text { NYHA classification/proxy available } \\
\text { NYHA Class I } \\
\text { NYHA Class II } \\
\text { NYHA Class III } \\
\text { NYHA Class IV }\end{array}$ & $\begin{array}{l}999(44.4) \\
1654(73.5) \\
458(20.3) \\
1354(60.2) \\
685(30.4) \\
1598(71.0) \\
1334(59.3) \\
537(40.3) \\
447(33.5) \\
299(22.4) \\
51(3.8)\end{array}$ & $\begin{array}{l}1638(30.7) \\
3285(61.6) \\
785(14.7) \\
2570(48.2) \\
824(15.5) \\
4013(75.3) \\
1562(29.3) \\
452(28.9) \\
530(33.9) \\
479(30.7) \\
101(6.5)\end{array}$ & $\begin{array}{l}<0.001 \\
<0.001 \\
<0.001 \\
<0.001 \\
<0.001 \\
<0.001 \\
<0.001\end{array}$ \\
\hline
\end{tabular}

Notes: Data are presented as $\mathrm{n}(\%)$ unless otherwise indicated. Comparisons made using $\chi^{2}$ test for categorical values and Mann-Whitney U-test for continuous or count variables. ${ }^{2}$ Patient percentages for $\mathrm{mMRC}$ are presented as row percentages; the other variables are calculated using column percentages. ${ }^{b}$ Smoking status was determined using the Read code closest to and $<5$ years before the index date ( 3 months after new HF diagnosis). ${ }^{C} m M R C$ score was defined as the Read code recorded closest to and $<5$ years before (or otherwise $<5$ years after) the index date. ${ }^{\mathrm{d}} \mathrm{GOLD}$ group was calculated using exacerbation and $\mathrm{mMRC}$ data recorded closest to and within 5 years before or after the index date $\left(2017 \mathrm{criteria}^{36}\right)$. ${ }^{\mathrm{e} F E V}$, determined closest to and within 5 years before the index date. ${ }^{\mathrm{f}} \mathrm{NH}$ A classification determined by Read code closest to and within 5 years before the index date. NYHA data were limited in primary care medical records so breathlessness Read codes were used as proxies for NYHA class.

Abbreviations: $\mathrm{CCl}$, Charlson comorbidity index; GERD, gastroesophageal reflux disease; GOLD, Global initiative for chronic Obstructive Lung Disease; IHD, ischemic heart disease; IQR, interquartile range; mMRC, modified Medical Research Council dyspnea scale; n/a, not available; NYHA, New York Heart Association; script, prescription. 


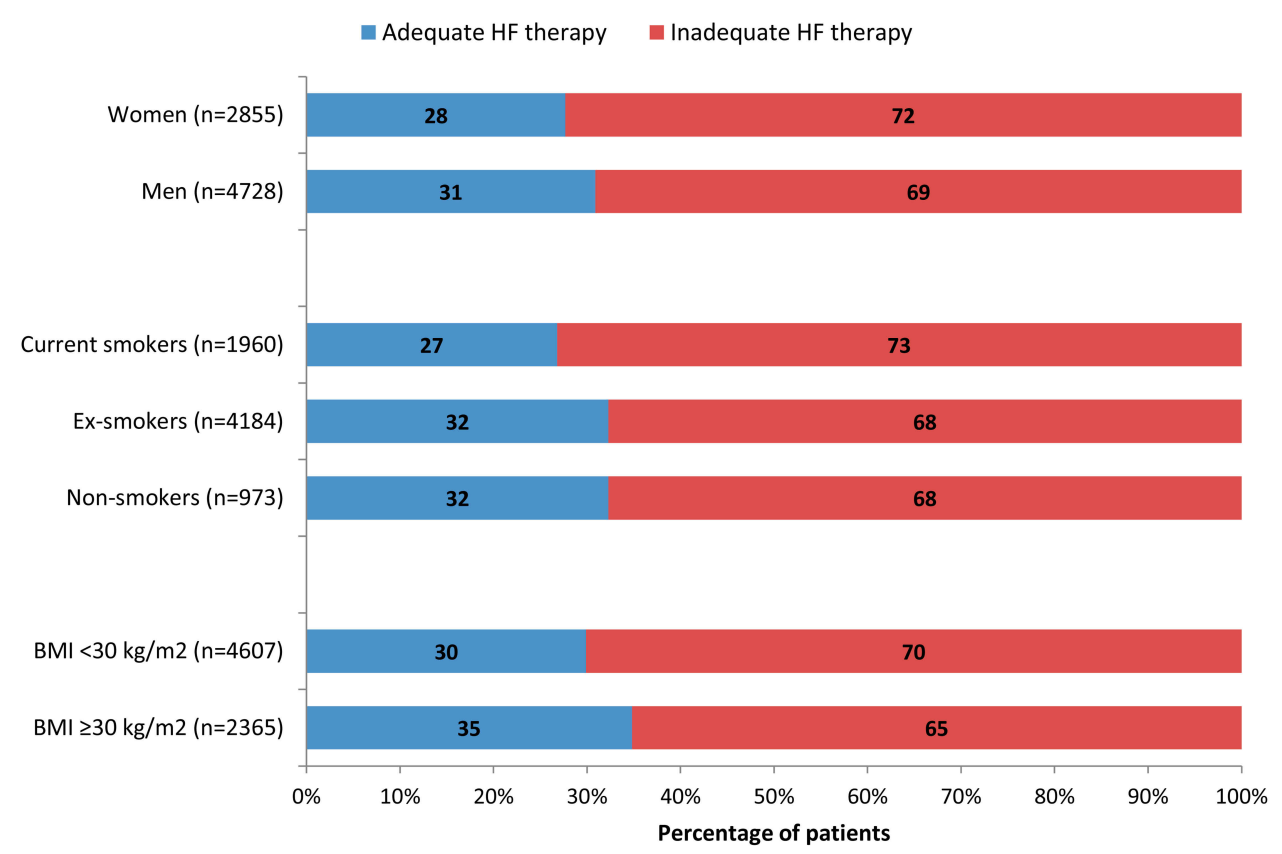

Figure 4 Sex, smoking status, and body mass index categories of patients with pre-existing COPD who were prescribed adequate versus inadequate heart failure (HF) therapy within 3 months of a new HF diagnosis (COPD-new HF cohort). $\chi^{2}$ test $p=0.003$ for comparisons between women and men; $p<0.00 \mathrm{I}$ for comparisons among smoking status categories and between BMI categories.

secondary care. ${ }^{43}$ Interestingly, we found that a greater percentage of patients in both adequate therapy groups, as compared with the inadequate therapy groups, had other common comorbidities, including gastroesophageal reflux disease, diabetes, hypertension, and chronic renal disease. We do not have definitive explanations for this finding, although the higher rate of adequate therapy for these patients may reflect a multidisciplinary management of patients with multimorbidity. Many unanswered questions remain regarding optimal therapy for and reasons for under-treatment of comorbid COPD and HF in general practice. ${ }^{44}$

The strengths of this descriptive study include the use of long-term longitudinal data from quality-controlled UK databases frequently used for pharmacoepidemiologic research. ${ }^{7,45,46}$ These findings illustrate real-life prescribing patterns for large, representative patient cohorts in the UK. Moreover, the institution of the Quality and Outcomes Framework in 2004 provides incentives for recording of key disease-related measures in primary care EMRs, which has improved the recording of variables such as smoking status, recorded for $>93 \%$ of patients in each cohort. ${ }^{34}$ Quint et al $^{45}$ studied the accuracy of COPD diagnoses in the CPRD from 2004 to 2012 and found that COPD diagnostic codes accurately identified patients with COPD, with only marginally improved accuracy if recorded spirometry or prescribed COPD medications were used to confirm the COPD diagnosis. The median index date years were later for the adequate therapy groups, possibly suggesting improvements in prescribing practices over time that may have been led by changes in diagnostic criteria, guidelines, and the availability of newer treatments.

The CPRD and OPCRD data are recorded for clinical and routine use, however, rather than specifically for research purposes. Whilst smoking status was recorded for the majority of patients, the proportion of missing data for some patient groups was high for certain variables. For example, $\mathrm{FEV}_{1}$ data was not available for $65 \%$ of patients in the inadequate therapy group (vs $43 \%$ of the adequate therapy group) of the HF-new COPD cohort, and $\mathrm{FEV}_{1}$ and $\mathrm{mMRC}$ data were missing for $61 \%$ and $32 \%$ of patients, respectively, in the inadequate therapy group (vs $35 \%$ and $13 \%$, respectively, of the adequate therapy group) of the COPD-new HF cohort. Moreover, there are limitations to the use of EMR data, as not all patients had definitive tests; as noted, many were missing spirometry results, and not all would have had an echocardiogram. Spirometry results that were recorded as free text and not entered as coded data were not included in our data, which could at least partially explain data missingness. Other study limitations are that we applied the GOLD 2017 criteria to evaluate prescribing practices before 2017, and we used only the mMRC to determine GOLD groups, as 
COPD assessment test results were not included in these earlier EMR data.

Some people may have been misdiagnosed as having HF and later correctly diagnosed as having COPD; however, the retrospective study design does not allow us to disentangle this possibility. In addition, as information on left ventricular ejection fraction was lacking, we could not separate HF with reduced ejection fraction from $\mathrm{HF}$ with preserved ejection fraction, a relevant distinction because, for the latter, evidence is lacking or inconsistent with regard to the benefits of beta-blockers, ACE inhibitors, and ARBs. ${ }^{23}$

We acknowledge that the study period was lengthy (1988 through May 2015) and thus incorporated changes in guidelines and prescribing practices over time, although newer therapies were not yet in recommended use. However, the majority of diagnoses were much later than 1988, with median diagnosis years of 2004 for inadequate treatment groups and 2009-2010 for adequate treatment groups. Moreover, whilst we did not assess drug doses, any occurrence of under-dosing would increase the incidence of treatment inadequacy. Information about the use of non-pharmacological treatments, such as rehab and smoking cessation aids, was not available in the CPRD and OPCRD. Finally, as for all database studies, there is the possibility of errors and omissions in recorded data.

Because of the multiple differences between adequate and inadequate therapy groups, matched analyses and/or adjusted models would be required to conduct a proper statistical comparison between these two groups to account for these differences. However, the process of matching the adequately to inadequately treated patients would likely result in a large number of patients being excluded, greatly reducing the generalizability of the results. Furthermore, given the large number of candidate characteristics that could be included, and the size of baseline differences, the process of developing adjusted models would be complex and may not produce robust results. It would also be difficult to provide meaningful clinical interpretation of these models because of the large baseline differences we identified.

Further studies are needed to understand the reasons for the under-prescribing identified by this study as well as by others. ${ }^{25,26,39}$ Longitudinal analysis techniques would be useful in a future study to better understand temporal differences in prescribing as relates to guideline adherence and drug availability. This would illustrate how treatment has changed over time and highlight any current treatment disparities that remain to be addressed. Moreover, work is needed to provide clinical education to improve and maximize appropriate prescribing for all patient subgroups, as well as to encourage broader use of spirometric testing when COPD is suspected. The optimal diagnostic and therapeutic approaches to comorbid COPD and HF require elucidation and implementation, as outlined in a recent call for action. ${ }^{47}$ To that end, Roversi et al $^{17}$ have proposed diagnostic and treatment algorithms for patients suspected of having comorbid COPD and HF that could be adapted for wider dissemination.

Other important areas for further study are to determine why clinicians may be reluctant to prescribe beta-blockers, as well as whether there are actual benefits of treatment with beta-blockers, in patients with COPD who have HF. ${ }^{48}$ The results of observational studies indicate there may be beneficial effects of beta-blockers for patients with COPD, ${ }^{49-51}$ and this question is being further explored in a prospective randomized trial. ${ }^{52}$ Of note, in a recent placebo-controlled trial of metoprolol for patients with COPD at increased risk of exacerbation-that specifically excluded patients with established indications for a betablocker - the risk of exacerbation was not lowered in the metoprolol group. ${ }^{53}$ Finally, the safety of combined LABA and LAMA for patients with comorbid COPD and HF also requires further study. ${ }^{54,55}$

\section{Conclusion}

Many patients with comorbid COPD and HF receive inadequate therapy after a new diagnosis of both conditions. Our findings identify which patients are most likely to be prescribed inadequate COPD therapy, including women, current smokers, and non-obese patients, or inadequate HF therapy, including current smokers and nonobese patients, after a new diagnosis in real-life clinical practice. These findings support the need for improving the equity of access to integrated care for all patients with comorbid COPD and HF. This information could be used to develop future guidelines to aid clinicians in making treatment decisions for patients with these comorbid conditions. A routine recommendation to examine for these common comorbidities, and to provide adequate therapy for both, should be addressed and expanded in COPD as well as HF management guidelines.

\section{Acknowledgments}

We thank Derek Skinner for his contributions to the data acquisition and handling and Carole Nicholls and Priyanka Raju Konduru for statistical support. Writing and editorial 
support was provided by Elizabeth V. Hillyer, DVM, supported by Novartis Pharma AG, Basel, Switzerland.

\section{Funding}

This work was supported by Novartis. Employees of the sponsor (listed as authors) participated in the study design, interpretation of the results, writing of the report, and the decision to submit the paper for publication.

\section{Disclosure}

KK is now affiliated with the University of Ioannina Medical School, Ioannina, Greece and was an employee of Novartis Pharma AG at the time of the study; he has received consulting/ lecture fees from AstraZeneca, Boehringer Ingelheim, Chiesi, ELPEN, GSK, Menarini, Novartis, Sanofi, and NuvoAir. He also reports that his current department has received funding and grants from AstraZeneca, Boehringer Ingelheim, Chiesi, Innovis, ELPEN, GSK, Menarini, Novartis and NuvoAir. CKR received consulting/lecture fees from MSD, AstraZeneca, GSK, Novartis, Takeda, Mundipharma, Boehringer-Ingelheim, Teva, and Bayer. JRH received consulting/lecture fees (and to his employer), support to attend meetings and grant support from pharmaceutical companies that make medicines to treat COPD. PA reports personal fees from Menarini, Novartis, and Boehringer, and grants from Daiichi Sankyo, outside the submitted work. HC and RF are employees of Novartis Pharmaceuticals Corporation. RJ reports grants and personal fees (and to his employer) from AstraZeneca, Novartis and GlaxoSmithKline; personal fees from Boehringer Ingelheim, Chiesi, Nutricia, OPRI, and Pfizer. JWHK reports grants, personal fees and non-financial support from AstraZeneca, grants, personal fees and nonfinancial support from Boehringer Ingelheim, grants and personal fees from Chiesi Pharmaceuticals, grants, personal fees and non-financial support from GSK, personal fees from OPRI, grants and personal fees from Novartis, grants from MundiPharma, grants from TEVA, outside the submitted work. All personal fees are paid to the institutions. KM is an employee of Novartis Pharma AG. SWYM and R. Ryan were employees of the Observational and Pragmatic Research Institute (OPRI) at the time of the study. DBP has board membership with Amgen, AstraZeneca, Boehringer Ingelheim, Chiesi, Circassia, Mylan, Mundipharma, Napp, Novartis, Regeneron Pharmaceuticals, Sanofi Genzyme, Teva Pharmaceuticals; consultancy agreements with Amgen, AstraZeneca, Boehringer Ingelheim, Chiesi, GlaxoSmithKline, Mylan, Mundipharma, Napp, Novartis, Pfizer, Teva Pharmaceuticals, Theravance; grants and unrestricted funding for investigator-initiated studies (conducted through Observational and Pragmatic Research Institute Pte Ltd) from AKL Research and Development Ltd, AstraZeneca, Boehringer Ingelheim, British Lung Foundation, Chiesi, Circassia, Mylan, Mundipharma, Napp, Novartis, Pfizer, Regeneron Pharmaceuticals, Respiratory Effectiveness Group, Sanofi Genzyme, Teva Pharmaceuticals, Theravance, UK National Health Service, Zentiva (Sanofi Generics); payment for lectures/speaking engagements from AstraZeneca, Boehringer Ingelheim, Chiesi, Cipla, GlaxoSmithKline, Kyorin, Mylan, Merck, Mundipharma, Novartis, Pfizer, Regeneron Pharmaceuticals, Sanofi Genzyme, Teva Pharmaceuticals; payment for manuscript preparation from Mundipharma, Teva Pharmaceuticals; payment for the development of educational materials from Mundipharma, Novartis; payment for travel/accommodation/meeting expenses from AstraZeneca, Boehringer Ingelheim, Circassia, Mundipharma, Napp, Novartis, Teva Pharmaceuticals; funding for patient enrolment or completion of research from Chiesi, Novartis, Teva Pharmaceuticals, Zentiva (Sanofi Generics); stock/stock options from AKL Research and Development Ltd which produces phytopharmaceuticals; owns $74 \%$ of the social enterprise Optimum Patient Care Ltd (Australia and UK) and $74 \%$ of Observational and Pragmatic Research Institute Pte Ltd (Singapore); and is peer reviewer for grant committees of the Efficacy and Mechanism Evaluation programme, and Health Technology Assessment. The authors report no other conflicts of interest in this work.

\section{References}

1. Academy of Medical Sciences. Multimorbidity: a priority for global health research; 2018. Available from: https://acmedsci.ac.uk/policy/ policy-projects/multimorbidity. Accessed April 17, 2020.

2. The Lancet. Making more of multimorbidity: an emerging priority. Lancet. 2018;391(10131):1637. doi:10.1016/S0140-6736(18)30941-3

3. Vanfleteren LE, Spruit MA, Franssen FM. Tailoring the approach to multimorbidity in adults with respiratory disease: the NICE guideline. Eur Respir J. 2017;49(2):1601696. doi:10.1183/13993003.01696-2016

4. Global initiative for chronic Obstructive Lung Disease (GOLD). Global strategy for prevention, diagnosis and management of COPD; 2020. Available from: https://goldcopd.org/gold-reports/. Accessed April 17, 2020.

5. Vogelmeier CF, Criner GJ, Martinez FJ, et al. Global strategy for the diagnosis, management, and prevention of chronic obstructive lung disease 2017 report: GOLD executive summary. Eur Respir J. 2017;49 (3): 1700214. doi:10.1183/13993003.00214-2017

6. Chen W, Thomas J, Sadatsafavi M, FitzGerald JM. Risk of cardiovascular comorbidity in patients with chronic obstructive pulmonary disease: a systematic review and meta-analysis. Lancet Respir Med. 2015;3(8):631-639. doi:10.1016/S2213-2600(15)00241-6

7. Morgan AD, Rothnie KJ, Bhaskaran K, Smeeth L, Quint JK. Chronic obstructive pulmonary disease and the risk of 12 cardiovascular diseases: a population-based study using UK primary care data. Thorax. 2018;73(9):877-879. doi:10.1136/thoraxjnl-2017-210865 
8. Hawkins NM, Petrie MC, Jhund PS, Chalmers GW, Dunn FG, McMurray JJ. Heart failure and chronic obstructive pulmonary disease: diagnostic pitfalls and epidemiology. Eur J Heart Fail. 2009;11 (2):130-139. doi:10.1093/eurjhf/hfn013

9. Rabe KF, Hurst JR, Suissa S. Cardiovascular disease and COPD: dangerous liaisons? Eur Respir Rev. 2018;27(149):180057. doi:10.1183/16000617.0057-2018

10. Sidney S, Sorel M, Quesenberry CP, DeLuise C, Lanes S, Eisner MD. COPD and incident cardiovascular disease hospitalizations and mortality: kaiser permanente medical care program. Chest. 2005;128 (4):2068-2075. doi:10.1378/chest.128.4.2068

11. Huiart L, Ernst P, Suissa S. Cardiovascular morbidity and mortality in COPD. Chest. 2005;128(4):2640-2646. doi:10.1378/chest.128.4.2640

12. Fisher KA, Stefan MS, Darling C, Lessard D, Goldberg RJ. Impact of COPD on the mortality and treatment of patients hospitalized with acute decompensated heart failure: the Worcester Heart Failure Study. Chest. 2015;147(3):637-645. doi:10.1378/chest.14-0607

13. Canepa M, Temporelli PL, Rossi A, et al. Prevalence and prognostic impact of chronic obstructive pulmonary disease in patients with chronic heart failure: data from the GISSI-HF trial. Cardiology. 2017;136(2):128-137. doi:10.1159/000448166

14. Divo M, Cote C, de Torres JP, et al. Comorbidities and risk of mortality in patients with chronic obstructive pulmonary disease. Am J Respir Crit Care Med. 2012;186(2):155-161. doi:10.1164/rccm.201201-0034OC

15. Boudestein LC, Rutten FH, Cramer MJ, Lammers JW, Hoes AW. The impact of concurrent heart failure on prognosis in patients with chronic obstructive pulmonary disease. Eur J Heart Fail. 2009;11 (12):1182-1188. doi:10.1093/eurjhf/hfp148

16. Hawkins NM, Virani S, Ceconi C. Heart failure and chronic obstructive pulmonary disease: the challenges facing physicians and health services. Eur Heart J. 2013;34(36):2795-2803. doi:10.1093/eurheartj/eht192

17. Roversi S, Fabbri LM, Sin DD, Hawkins NM, Agusti A. Chronic obstructive pulmonary disease and cardiac diseases: an urgent need for integrated care. Am J Respir Crit Care Med. 2016;194 (11):1319-1336. doi:10.1164/rccm.201604-0690SO

18. Güder G, Störk S. COPD and heart failure: differential diagnosis and comorbidity. Herz. 2019;44(6):502-508. doi:10.1007/s00059-019-4814-7

19. Roversi S, Boschetto P, Beghe B, et al. Breathlessness, but not cough, suggests chronic obstructive pulmonary disease in elderly smokers with stable heart failure. Multidiscip Respir Med. 2018;13(1):35. doi:10.1186/s40248-018-0148-1

20. National Institute for Health and Care Excellence (NICE). Chronic obstructive pulmonary disease in over 16s: diagnosis and management: NICE guideline [NG115]; 2019. Available from: https://www. nice.org.uk/guidance/ng115. Accessed April 17, 2020.

21. National Institute for Health and Care Excellence (NICE). Chronic heart failure in adults: diagnosis and management: NICE guideline [NG106]; 2018. Available from: https://www.nice.org.uk/guidance/ ng106. Accessed April 17, 2020.

22. National Institute for Health and Care Excellence (NICE). Acute heart failure: diagnosis and management: clinical guideline [CG187]; 2014. Available from: https://www.nice.org.uk/guidance/ cg187. Accessed April 17, 2020.

23. Ponikowski P, Voors AA, Anker SD, et al. ESC guidelines for the diagnosis and treatment of acute and chronic heart failure: the task force for the diagnosis and treatment of acute and chronic heart failure of the European Society of Cardiology (ESC). Developed with the special contribution of the Heart Failure Association (HFA) of the ESC. Eur Heart J. 2016;37(27):2129-2200. doi:10.1093/eurheartj/ehw128

24. Dickstein K, Cohen-Solal A, Filippatos G, et al. ESC guidelines for the diagnosis and treatment of acute and chronic heart failure 2008: the task force for the diagnosis and treatment of acute and chronic heart failure 2008 of the European Society of Cardiology. Developed in collaboration with the Heart Failure Association of the ESC (HFA) and endorsed by the European Society of Intensive Care Medicine (ESICM). Eur Heart J. 2008;29(19):2388-2442. doi:10.1093/eurheartj/ehn309
25. Lipworth B, Skinner D, Devereux G, et al. Underuse of $\beta$-blockers in heart failure and chronic obstructive pulmonary disease. Heart. 2016;102(23):1909-1914. doi:10.1136/heartjnl-2016-309458

26. Girouard C, Gregoire JP, Poirier P, Moisan J. Factors associated with beta-blocker initiation and discontinuation in a population-based cohort of seniors newly diagnosed with heart failure. Patient Prefer Adherence. 2016;10:1811-1821.

27. UK Medicines and Healthcare products Regulatory Agency and the National Institute for Health Research (NIHR). Clinical Practice Research Datalink (CPRD). Available from: http://www.cprd.com/ home/. Accessed April 17, 2020.

28. Optimum Patient Care Research Database (OPCRD). Available from: http://opcrd.co.uk/. Accessed May 17, 2020.

29. Jones RC, Price D, Ryan D, et al. Opportunities to diagnose chronic obstructive pulmonary disease in routine care in the UK: a retrospective study of a clinical cohort. Lancet Respir Med. 2014;2(4):267-276. doi:10.1016/S2213-2600(14)70008-6

30. ENCePP/European Union electronic Register of Post-Authorisation Studies (EU PAS Register). Evaluation of the undertreatment and disease outcomes for patients with coexisting Heart Failure and Chronic Obstructive Pulmonary Disease (EU PAS Register Number EUPAS12987); 2016. Available from: http://www.encepp.eu/encepp/ v i e w R e s o u r e . ht m; j s e s s i o n i d = AJ0cVeWqupkWwutjPaEh48dAOvHLSoJIi2fOczb8EbAMeNzG80v a!-186951307?id=12988. Accessed April 17, 2020.

31. Roche N, Reddel H, Martin R, et al. Quality standards for real-world research. Focus on observational database studies of comparative effectiveness. Ann Am Thorac Soc. 2014;11(Suppl 2):S99-S104. doi:10.1513/AnnalsATS.201309-300RM

32. Roche N, Campbell JD, Krishnan JA, et al. Quality standards in respiratory real-life effectiveness research: the REal Life EVidence AssessmeNt Tool (RELEVANT): report from the Respiratory Effectiveness Group-European Academy of Allergy and Clinical Immunology Task Force. Clin Transl Allergy. 2019;9(1):20. doi:10.1186/s13601-019-0255-x

33. NHS Digital. Read codes. Available from: https://igital.nhs.uk/services/ terminology-and-classifications/read-codes. Accessed April 17, 2020.

34. British Medical Association. Quality and outcomes framework (QOF). Available from: https://www.bma.org.uk/advice-and-support/gp-practices /funding-and-contracts/quality-and-outcomes-framework-qof. Accessed April 17, 2020.

35. National Institute for Health and Care Excellence (NICE). NICE quality and outcomes framework indicator. Available from: https:// www.nice.org.uk/standards-and-indicators/qofindicators. Accessed April 17, 2020.

36. Global initiative for chronic Obstructive Lung Disease (GOLD). Global strategy for diagnosis, management, and prevention of COPD; 2017. Available from: https://goldcopd.org/archived-reports/. Accessed April 17, 2020.

37. Gruffydd-Jones K, Brusselle G, Jones R, et al. Changes in initial COPD treatment choice over time and factors influencing prescribing decisions in UK primary care: a real-world study. NPJ Prim Care Respir Med. 2016;26(1):16002. doi:10.1038/npjpcrm.2016.2

38. Lawson CA, Mamas MA, Jones PW, et al. Association of medication intensity and stages of airflow limitation with the risk of hospitalization or death in patients with heart failure and chronic obstructive pulmonary disease. JAMA Netw Open. 2018;1(8):e185489. doi:10.1001/jamanetworkopen.2018.5489

39. Canepa M, Straburzynska-Migaj E, Drozdz J, et al. Characteristics, treatments and 1-year prognosis of hospitalized and ambulatory heart failure patients with chronic obstructive pulmonary disease in the European Society of Cardiology Heart Failure Long-Term Registry. Eur J Heart Fail. 2018;20(1):100-110. doi:10.1002/ejhf.964

40. Sessa M, Mascolo A, Mortensen RN, et al. Relationship between heart failure, concurrent chronic obstructive pulmonary disease and beta-blocker use: a Danish nationwide cohort study. Eur J Heart Fail. 2018;20(3):548-556. doi:10.1002/ejhf.1045 
41. Roversi S, Tonelli R, Beghe B, et al. Use of adjunct cardiovascular therapy in patients hospitalised for acute exacerbations of COPD. ERJ Open Res. 2018;4(3):00087-2018. doi:10.1183/23120541.00087-2018

42. Huang YL, Lai CC, Wang YH, et al. Impact of selective and nonselective beta-blockers on the risk of severe exacerbations in patients with COPD. Int J Chron Obstruct Pulmon Dis. 2017;12:2987-2996. doi:10.2147/COPD.S145913

43. Jones RC, Dickson-Spillmann M, Mather MJ, Marks D, Shackell BS. Accuracy of diagnostic registers and management of chronic obstructive pulmonary disease: the Devon primary care audit. Respir Res. 2008;9(1):62. doi:10.1186/1465-9921-9-62

44. Canepa M, Franssen FME, Olschewski H, et al. Diagnostic and therapeutic gaps in patients with heart failure and chronic obstructive pulmonary disease. JACC Heart Fail. 2019;7(10):823-833. doi:10.1016/j.jchf.2019.05.009

45. Quint JK, Mullerova H, DiSantostefano RL, et al. Validation of chronic obstructive pulmonary disease recording in the Clinical Practice Research Datalink (CPRD-GOLD). BMJ Open. 2014;4(7): e005540. doi:10.1136/bmjopen-2014-005540

46. Halpin DM, Kerkhof M, Soriano JB, Mikkelsen H, Price DB. Eligibility of real-life patients with COPD for inclusion in trials of inhaled long-acting bronchodilator therapy. Respir Res. 2016;17 (1):120. doi:10.1186/s12931-016-0433-5

47. Leitao Filho FS, Sin DD. COPD and cardiovascular diseases: now is the time for action! Thorax. 2018;73(9):799-800. doi:10.1136/thoraxjnl-2018-211553
48. Lipworth B, Wedzicha J, Devereux G, Vestbo J, Dransfield MT. Betablockers in COPD: time for reappraisal. Eur Respir J. 2016;48 (3):880-888. doi:10.1183/13993003.01847-2015

49. Bhatt SP, Wells JM, Kinney GL, et al. $\beta$-blockers are associated with a reduction in COPD exacerbations. Thorax. 2016;71(1):8-14. doi:10.1136/thoraxjnl-2015-207251

50. Short PM, Lipworth SI, Elder DH, Schembri S, Lipworth BJ. Effect of beta blockers in treatment of chronic obstructive pulmonary disease: a retrospective cohort study. BMJ. 2011;342(may10 2):d2549. doi:10.1136/bmj.d2549

51. Du Q, Sun Y, Ding N, Lu L, Chen Y. Beta-blockers reduced the risk of mortality and exacerbation in patients with COPD: a meta-analysis of observational studies. PLoS One. 2014;9(11):e113048. doi:10.1371/journal.pone.0113048

52. Bisoprolol in COPD study (BICS). 2018. Available from: http://www. isrctn.com/ISRCTN10497306. Accessed April 14, 2020.

53. Dransfield MT, Voelker H, Bhatt SP, et al. Metoprolol for the prevention of acute exacerbations of COPD. $N$ Engl J Med. 2019;381 (24):2304-2314. doi:10.1056/NEJMoa1908142

54. Agabiti N, Corbo GM. COPD and bronchodilators: should the heart pay the bill for the lung? Eur Respir J. 2017;49(5):1700370. doi:10.1183/13993003.00370-2017

55. Suissa S, Dell'Aniello S, Ernst P. Concurrent use of long-acting bronchodilators in COPD and the risk of adverse cardiovascular events. Eur Respir J. 2017;49(5):1602245. doi:10.1183/13993003.02245-2016

\section{Publish your work in this journal}

Pragmatic and Observational Research is an international, peerreviewed, open access journal that publishes data from studies designed to reflect more closely medical interventions in realworld clinical practice compared with classical randomized controlled trials (RCTs). The manuscript management system is completely online and includes a very quick and fair peer-review system. Visit http://www.dovepress.com/testimonials.php to read real quotes from published authors. 